\title{
Synthesis of new 2,3-disubstituted pyridines containing a 1,2,3-triazole in the side-chain via one-pot copper-catalyzed azide-alkyne cycloaddition
}

\author{
Meenakshi Verma, Vijay Luxami and Kamaldeep Paul* \\ School of Chemistry and Biochemistry, Thapar University, Patiala-147 001, India \\ E-mail:kpaul@thapar.edu
}

DOI: http://dx.doi.org/10.3998/ark.5550190.p009.175

\begin{abstract}
A series of 1,2,3-triazole-containing pyridines has been synthesized using the $\mathrm{Cu}$ (II) catalyzed 'click approach' from sodium azide and corresponding halides. The synthesis involves the amidation of 2-amino-3-hydroxypyridine with benzoyl chloride or cinnamic acid followed by reaction with propargyl bromide to obtain $N$-(3-(prop-2-ynyloxy)pyridin-2yl)benzamide 5 and 3-phenyl- $N$-(3-(prop-2-ynyloxy)-pyridin-2-yl)acrylamide 10 respectively. These compounds underwent one-pot tandem copper-catalyzed azidation and CuAAC reactions to provide compounds $\mathbf{6 a - h}$ and $\mathbf{1 1 a - g}$ in moderate to good yields.
\end{abstract}

Keywords: One-pot click chemistry, pyridine, 1,2,3-triazole, benzyl halides

\section{Introduction}

Due to their chemical and biological importance, heterocyclic moieties are attractive targets in medicinal and pharmaceutical chemistry. ${ }^{1-2}$ Among heterocyclic compounds, pyridine has proved to be the one of the most important moieties. Substituted pyridines have a major role in a wide variety of natural products, pharmaceutical drugs and various kinds of functional materials. ${ }^{3-4}$ For the synthesis of pyridines, various methods are known in the literature. John Spencer et al. ${ }^{5}$ synthesized libraries of pyridine derivatives, many of which contain a piperazine group at the 2-position. Sreekantha B. Jonnalagadda et al. ${ }^{6}$ synthesized highly substituted pyridines in good yields by the use of $\mathrm{Au}$ loaded $\mathrm{MgO}$ in one-pot, multicomponent system. Haider Behbehani et al. ${ }^{7}$ explored a series of 5-arylazo-2,3,6-trisubstituted pyridines from the reactions of 3-oxo-2-arylhydrazonopropanal with 3-oxo-3-phenylpropionitrile. Xie et al. ${ }^{8}$ synthesized 5,6-disubstituted pyridine-2,3-dione-3-thiosemicarbazone derivatives and 5,6disubstituted pyridine-2,3-dione $S$-benzyl-3-thiosemicarbazones via oxidation-Michael additions, condensations and nucleophilic substitutions.

$\mathrm{Cu}$-promoted coupling reactions have been extensively used for the synthesis of several molecules and biomolecules. ${ }^{9-11}$ The azidation reaction has been used as a facile method for the transformation of an aryl halide into an aryl azide. Synthetic applications of these ar- 
yl/alkyl azides have become highly attractive alternatives to establish nitrogen-linked aryl/alkyl scaffolds. This high yield and regioselective reaction has found numerous applications ranging from chemistry to biology. Copper-catalyzed azide-alkyne cycloaddition ( $\mathrm{Cu}-$ AAC) reaction (click chemistry) has been widely utilized for the synthesis of biologically active 1,2,3-triazole compounds. Several therapeutically active compounds containing this moiety have been reported, viz., anti-HIV agents, antimicrobials, and kinase inhibitors. ${ }^{12-16}$ Moreover, $\mathrm{Cu}$-promoted azidation and 1,3-dipolar [3 +2$]$ cycloaddition reactions between azides and terminal alkynes that can be carried out in one-pot synthesis are an attractive method. Herein, we demonstrate an efficient synthesis of novel 2,3-disubstituted pyridines by performing one-pot tandem copper-catalyzed azidation and $\mathrm{CuAAC}$ reaction.

\section{Results and Discussion}

In our present work, the precursor of $N$-(3-(prop-2-ynyloxy)-pyridin-2-yl)benzamide 5 was synthesized from an easily available starting material 2-amino-3-hydroxypyridine 1 (Scheme 1). Compound 1 was reacted with benzoyl chloride 2 in the presence of potassium carbonate and acetonitrile using tetrabutylammonium hydrogen sulfate $\left(\mathrm{TBAHSO}_{4}\right)$ as catalyst at reflux temperature for eight hours to give of $N$-(3-hydroxy-pyridin-2-yl)benzamide 3 and benzoic acid 2-amino-pyridin-3-yl ester 4 as white solids in $65 \%$ and $10 \%$ yields respectively. The crude 3 was $>95 \%$ pure according to ${ }^{1} \mathrm{H}$ NMR and was used in the next stage without further purification. The reaction of $\mathbf{3}$ with propargyl bromide and potassium carbonate in acetonitrile with $\mathrm{TBAHSO}_{4}$ proceeded at reflux temperature for eight hours to give $\mathbf{5}$ as a brown coloured liquid in $70 \%$ yield. The final step was the synthesis of pyridine analogues containing a 1,2,3-triazole unit 6a-h from $\mathbf{5}$ and various benzyl halides. A Cu-catalyzed click reaction was performed with $\mathbf{5}$ and benzyl chloride using sodium azide, copper(II) acetate as catalyst, sodium ascorbate as reducing agent and 1,10-phenanthroline. $\mathrm{H}_{2} \mathrm{O}$ as ligand in EtOH: $\mathrm{H}_{2} \mathrm{O}(6: 4)$ at room temperature for $24 \mathrm{~h}$ to afford the crude product. Addition of cold water to the reaction mixture resulted in formation of a white precipitate. The crude product was then subjected to column chromatography to afford $N$-(3-((1-benzyl-1H-1,2,3-triazol-4yl)methoxy)pyridin-2-yl)benzamide $\mathbf{6 a}$ in 55\% yield (Table 1, entry 1). Similarly, other 1,2,3triazoles (6b-h, Table 1, entry 2-8) were synthesized by the reaction of compound $\mathbf{5}$ with other substituted benzyl halides and allyl bromide in moderate to good yields (55-82\%). The structure of 6a was confirmed by ${ }^{1} \mathrm{H}$ and ${ }^{13} \mathrm{C}$ NMR analysis as well as mass spectrometry. In the ${ }^{1} \mathrm{H}$ NMR spectrum, the $\mathrm{C} 5$-proton of the triazole ring resonated at $\delta 7.57$, the protons of the $\mathrm{OCH}_{2}$ group appeared as a singlet at $\delta 5.50$ and the protons of the $\mathrm{NCH}_{2}$ group as a singlet at $\delta 5.29$, along with aromatic proton signals. In the ${ }^{13} \mathrm{C} \mathrm{NMR}$ spectra, the $\mathrm{OCH}_{2}$ carbon appeared at $\delta 62.7$, the $\mathrm{NCH}_{2}$ carbon appeared at $\delta 54.2$, thje $\mathrm{C} 5$-carbon of the triazole was at $\delta$ 123.1, and carbonyl group of the amide at $\delta 164.8$, along with other carbons. In EI-MS, peak appeared at $\mathrm{m} / \mathrm{z}=386.3$ for $\left(\mathrm{M}^{+}+1\right)$ ion of 6a. Higher yields of 1,2,3-triazoles were obtained when a fluorine atom was present in the benzyl moiety. 


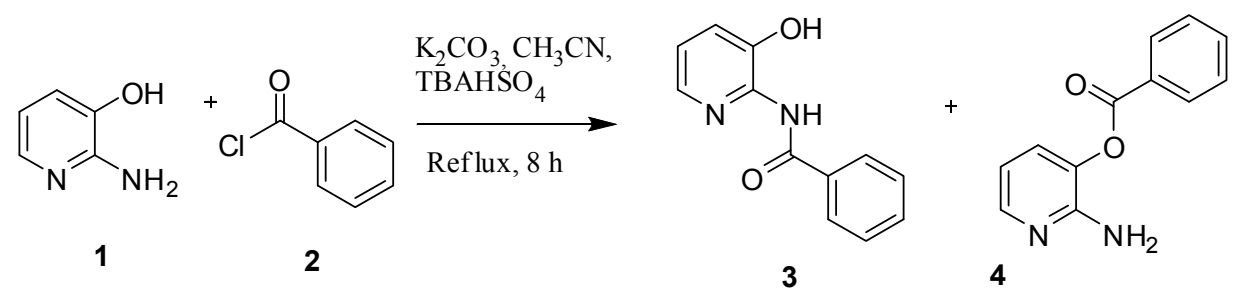<smiles>O=C(Nc1ncccc1O)c1ccccc1</smiles>

3

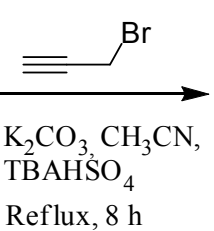

Reflux, 8 h<smiles>C#CCOc1cccnc1NC(=O)c1ccccc1</smiles>

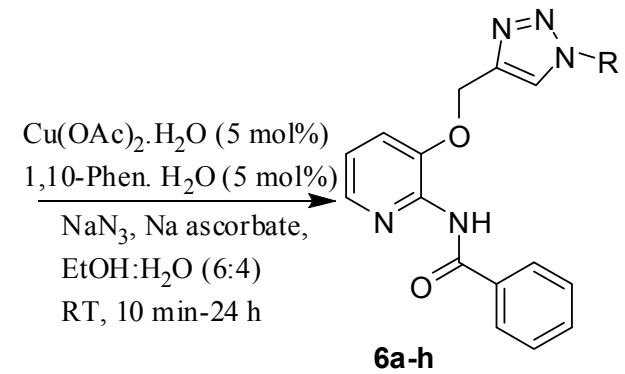

Scheme 1. Synthesis of $N$-(3-(1-substituted benzyl/allyl-1H-[1,2,3]-triazol-4-yl)methoxy) pyridine-2-yl)-3-phenyl-acrylamide

We also reacted 1 with cinnamic acid 7 in the presence of hydroxybenzotriazole (HOBt), $N$-(3-dimethylaminopropyl)- $N$ '-ethylcarbodiimide hydrochloride (EDC) and diisopropylethylamine (DIPEA) in dichloromethane at room temperature for 12 hours to yield $\mathrm{N}$-(3hydroxy-pyridin-2-yl)-3-phenyl-acrylamide 8 in $80 \%$ yield (Scheme 2). Compound 8 was then further treated with propargyl bromide in potassium carbonate and acetonitrile using $\mathrm{TBAHSO}_{4}$ as catalyst at room temperature for eight hours to give 3-phenyl- $N$-prop-2-ynyl$N$-(3-prop-2-ynyloxy-pyridin-2-yl)acrylamide $\quad 9 \quad$ and 3-phenyl- $N$-(3-(prop-2-ynyloxy)pyridin-2-yl)acrylamide 10 in 10\% and 55\% yields respectively. The same reaction conducted at reflux temperature gave compound $\mathbf{9}$ in $60 \%$ yield along with traces of compound $\mathbf{1 0}$.

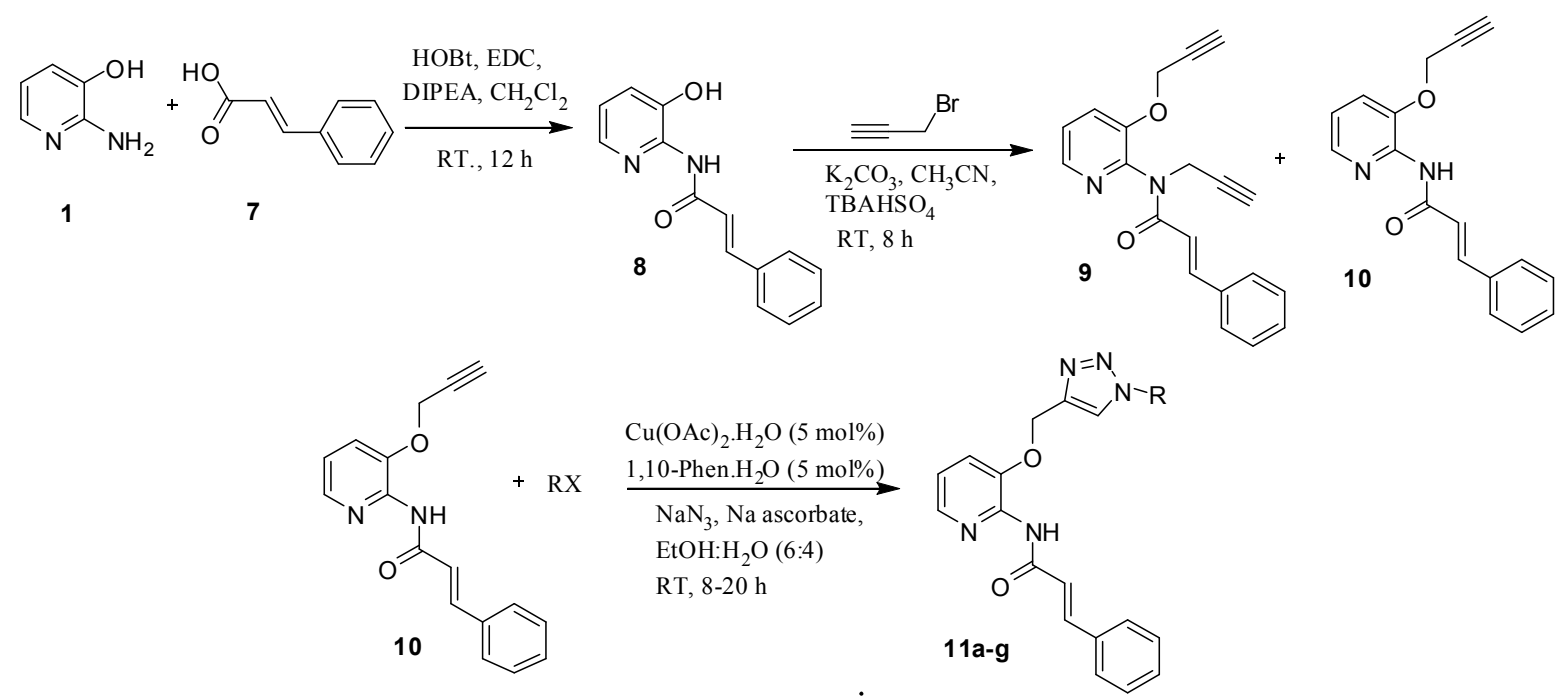

Scheme 2. Synthesis of $N$-(3-(1-substituted benzyl/allyl-1H-[1,2,3]-triazol-4-yl)methoxy) pyridine-2-yl)-3-phenyl-acrylamide 
Compound 10 underwent a copper-catalyzed click reaction with benzyl chloride using sodium azide in the presence of copper(II) acetate, sodium ascorbate and 1,10phenanthroline. $\mathrm{H}_{2} \mathrm{O}$ in EtOH: $\mathrm{H}_{2} \mathrm{O}$ at room temperature for 20 hours. Addition of cold water to the reaction mixture resulted in a white precipitatesof crude product which was then purified by column chromatography to give $N$-[3-(1-benzyl-1H-[1,2,3]triazol-4-ylmethoxy)pyridin-2-yl]-3-phenyl-acrylamide 11a in 50\% yield (Table 1, entry 9). In the ${ }^{1} \mathrm{H}$ NMR spectrum of this compound there was a singlet at $\delta 7.59$ for the $\mathrm{C} 5$-proton of the triazole ring, proton signals for the $\mathrm{OCH}_{2}$ at $\delta 5.64$, for the $\mathrm{NCH}_{2}$ at $\delta 5.30$ and signals for other protons. In the ${ }^{13} \mathrm{C}$ NMR of $11 \mathrm{a}$, the $\mathrm{C} 5$ carbon of the triazole ring resonated at $\delta 123.2$, the $\mathrm{OCH}_{2}$ carbon resonated at $\delta 62.4$, the $\mathrm{NCH}_{2}$ carbon at $\delta 54.3$ and amide carbonyl group carbon resonated at $\delta 164.8$, along with signals for the other carbons. In EI-MS, a peak appeared at $\mathrm{m} / \mathrm{z}=412.2$ for $\left(\mathrm{M}^{+}+1\right)$ ion of 11a. An array of novel triazole derivatives 11a-g was synthesized by varying the substitution on the benzyl grojup as well as allyl groups (Table 1, entry 9-15). The overall yields of compounds $\mathbf{6}$ and $\mathbf{1 1}$ were very good and the method is high yielding, simple, convenient and general. Structures of all newly synthesized compounds were characterized by ${ }^{1} \mathrm{H}$ and ${ }^{13} \mathrm{C} \mathrm{NMR}$ as well as mass spectrometry (Supporting Information).

Table 1. Physical data of compounds $6 \mathbf{a}-\mathbf{h}$ and $11 \mathbf{a}-\mathbf{g}$

\begin{tabular}{|c|c|c|c|c|c|c|}
\hline Entry & Compds. & $\mathrm{RX}$ & Product & Time & Yield $(\%)$ & $\mathrm{mp}\left({ }^{\circ} \mathrm{C}\right)$ \\
\hline 1 & $6 a$ & & & $24 \mathrm{~h}$ & 55 & $120-122$ \\
\hline 2 & $6 b$ & & & $20 \mathrm{~h}$ & 65 & $163-164$ \\
\hline 3 & $6 c$ & & & $20 \mathrm{~h}$ & 66 & $154-157$ \\
\hline 4 & 6d & & & $20 \mathrm{~h}$ & 64 & $125-126$ \\
\hline
\end{tabular}


Table 1 (continued)

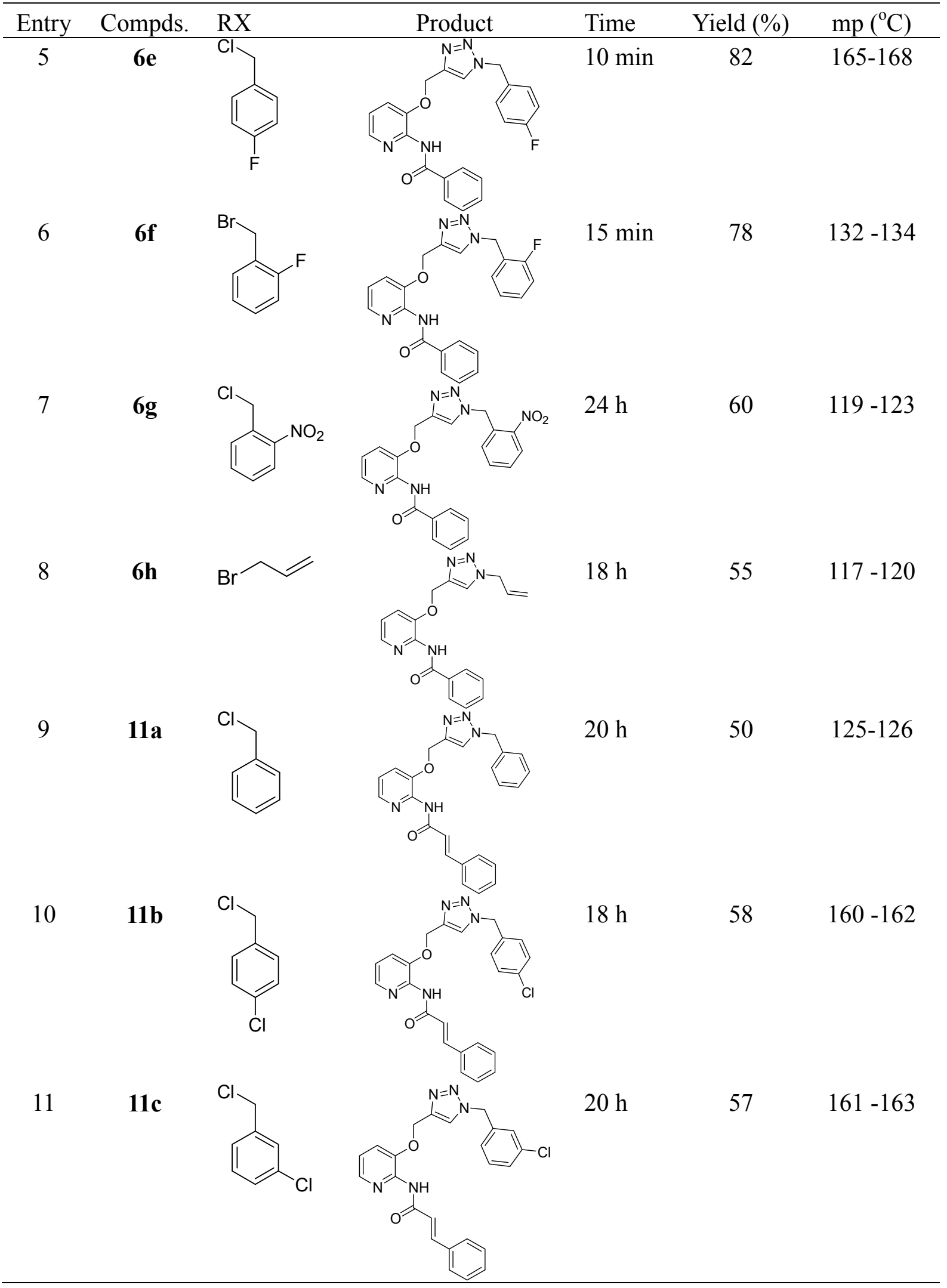


Table 1 (continued)

Entry Compds.

\section{Conclusions}

An efficient and straightforward one pot copper catalyzed azidation and $\mathrm{CuAAC}$ reaction for constructing novel 1,2,3-triazole-containing pyridines has been developed. Utilizing easily available reaction materials, a small library of pyridine derivatives carrying side-chain 1,2,3triazoles was rapidly and efficiently synthesized. Overall, we believe that the developed reaction method and novel series of pyridine substituted triazine should be considered as an important advance in medicinal and pharmaceutical chemistry.

\section{Experimental Section}

General. All chemicals and solvents were of commercial grade and used without further purification, supplied by spectrochemicals and Sigma-Aldrich. Melting points were determined in open capillaries and are uncorrected. ${ }^{1} \mathrm{H}$ and ${ }^{13} \mathrm{C}$ NMR spectra were recorded on Jeol ECS- 
$400 \mathrm{MHz}$ spectrometer at $400 \mathrm{MHz}$ and $100 \mathrm{MHz}$ respectively, using $\mathrm{CDCl}_{3}$ as solvent. The chemical shifts are expressed in parts per million with TMS as internal reference and $J$ values are given in Hz. Mass spectra were recorded on a Waters Micromass Q-Tof Micro (Milford, MA). Reactions were monitored by thin layer chromatography (TLC) with plates coated with silica gel HF-254. Column chromatography was performed with silica gel mesh size 60-120. Hexane: ethylacetate and ethyl acetate: methanol were used as solvent systems.

Synthesis of $\boldsymbol{N}$-(3-hydroxypyridin-2-yl)benzamide (3) and benzoic acid 2-aminopyridin3-yl ester (4). 2-Amino-3-hydroxy pyridine I (2 g, $18.17 \mathrm{mmol})$ was dissolved in acetonitrile $(50 \mathrm{~mL}), \mathrm{K}_{2} \mathrm{CO}_{3}(3 \mathrm{~g}, 21.75 \mathrm{mmol})$ and $\mathrm{TBAHSO}_{4}(0.05 \mathrm{mmol})$. Benzoyl chloride 2 (3 g, $21.75 \mathrm{mmol}$ ) was added and the reaction mixture was heated to reflux for $8 \mathrm{~h}$. The reaction mixture was neutralized with $\mathrm{NaHCO}_{3}$ and extracted with chloroform, the extract dried over $\mathrm{Na}_{2} \mathrm{SO}_{4}$, filtered and concentrated to get the crude product. The crude residue was then purified by column chromatography to get pure $N$-(3-hydroxy-pyridin-2-yl)benzamide (3) and benzoic acid 2-amino-pyridin-3-yl ester (4).

$\mathrm{N}$-(3-hydroxypyridin-2-yl)benzamide (3). Yield: $65 \%$; mp $130-133{ }^{\circ} \mathrm{C} ;{ }^{1} \mathrm{H}$ NMR (400 $\left.\mathrm{MHz}, \mathrm{CDCl}_{3}\right): \delta_{\mathrm{H}} 8.62(\mathrm{bs}, 1 \mathrm{H}, \mathrm{NH}), 8.34\left(\mathrm{dd},{ }^{2} J_{(\mathrm{HH})} 4.80 \mathrm{~Hz},{ }^{3} J_{(\mathrm{HH})} 1.48 \mathrm{~Hz}, 1 \mathrm{H}, \mathrm{H}-6\right), 8.16$ (dd, $\left.{ }^{2} J_{(\mathrm{HH})} 8.16 \mathrm{~Hz},{ }^{3} J_{(\mathrm{HH})} 0.96 \mathrm{~Hz}, 2 \mathrm{H}, \mathrm{H}-2^{\prime}, 6^{\prime}\right), 7.83$ (d, ${ }^{3} J_{(\mathrm{HH})} 7.04 \mathrm{~Hz}, 2 \mathrm{H}, \mathrm{H}-3^{\prime}, 5^{\prime}$ ), 7.77 $\left(\mathrm{dd},{ }^{2} J_{(\mathrm{HH})} 4.8 \mathrm{~Hz},{ }^{3} J_{(\mathrm{HH})} 8.08 \mathrm{~Hz}, 1 \mathrm{H}, \mathrm{H}-4\right.$ '), 7.60 (t, $\left.{ }^{3} J_{(\mathrm{HH})} 1.16 \mathrm{~Hz}, 1 \mathrm{H}, \mathrm{H}-4\right), 7.40$ (dd, ${ }^{2} J_{(\mathrm{HH})}$ $\left.7.32 \mathrm{~Hz},{ }^{3} J_{(\mathrm{HH})} 5.67 \mathrm{~Hz}, 1 \mathrm{H}, \mathrm{H}-5\right)$; MS (EI) : $m / z 215.1\left(\mathrm{M}^{+}+1\right)$.

Benzoic acid 2-aminopyridin-3-yl ester (4). Yield: 10\%; mp 160-162 ${ }^{\circ} \mathrm{C}$; ${ }^{1} \mathrm{H}$ NMR (400 $\mathrm{MHz}, \mathrm{CDCl}_{3}$ ): $\delta_{\mathrm{H}} 9.87$ (bs, 2H, NH $\left.\mathrm{N}_{2}\right), 8.02\left(\mathrm{dd},{ }^{2} J_{(\mathrm{HH})} 4.56 \mathrm{~Hz},{ }^{3} J_{(\mathrm{HH})} 1.48 \mathrm{~Hz}, 2 \mathrm{H}, \mathrm{H}-6,2^{\prime}\right)$, $7.62\left(\mathrm{t},{ }^{3} J_{(\mathrm{HH})} 1.20 \mathrm{~Hz}, 1 \mathrm{H}, \mathrm{H}-6\right)$ ) $7.58\left(\mathrm{t},{ }^{3} J_{(\mathrm{HH})} 7.88 \mathrm{~Hz}, 1 \mathrm{H}, \mathrm{H}-4\right), 7.51\left(\mathrm{t},{ }^{3} J_{(\mathrm{HH})} 7.88 \mathrm{~Hz}\right.$, $2 \mathrm{H}, \mathrm{H}-3$ ' , 5'), $7.41\left(\mathrm{dd},{ }^{2} J_{(\mathrm{HH})} 8.08 \mathrm{~Hz},{ }^{3} J_{(\mathrm{HH})} 1.48 \mathrm{~Hz}, 1 \mathrm{H}, \mathrm{H}-5\right), 7.11\left(\mathrm{dd},{ }^{2} J_{(\mathrm{HH})} 8.08 \mathrm{~Hz},{ }^{3} J_{(\mathrm{HH})}\right.$ $\left.4.64 \mathrm{~Hz}, 1 \mathrm{H}, \mathrm{H}-4^{\prime}\right)$; MS (EI) : $m / z 215.1\left(\mathrm{M}^{+}+1\right)$.

Synthesis of $\mathrm{N}$-(3-(prop-2-ynyloxy)pyridin-2-yl)benzamide (5). $\mathrm{N}$-(3-Hydroxy-pyridin-2yl)benzamide 3 ( $2 \mathrm{~g}, 10 \mathrm{mmol})$ was refluxed with propargyl bromide $(1.17 \mathrm{~g}, 10 \mathrm{mmol})$ in the presence of $\mathrm{K}_{2} \mathrm{CO}_{3}(2 \mathrm{~g}, 15 \mathrm{mmol})$ and $\mathrm{TBAHSO}_{4}(0.05 \mathrm{mmol})$ using acetonitrile as solvent for $8 \mathrm{~h}$. Acetonitrile was removed under vacuum, $\mathrm{CHCl}_{3}$ was added to it and the solution washed with water. The organic layer was dried over $\mathrm{Na}_{2} \mathrm{SO}_{4}$, filtered and concentrated to give the crude product. The crude product was purified by column chromatography to produce a sticky brown liquid of compound $\mathbf{5}$.

$\mathrm{N}$-(3-(prop-2-ynyloxy)pyridin-2-yl)benzamide (5). Yield: $70 \%$; ${ }^{1} \mathrm{H}$ NMR $(400 \mathrm{MHz}$, $\left.\mathrm{CDCl}_{3}\right): \delta_{\text {н }} 8.72(\mathrm{bs}, 1 \mathrm{H}, \mathrm{NH}), 8.16\left(\mathrm{dd},{ }^{2} J_{(\mathrm{HH})} 4.60 \mathrm{~Hz},{ }^{3} J_{(\mathrm{HH})} 1.36 \mathrm{~Hz}, 1 \mathrm{H}, \mathrm{H}-6\right), 7.94(\mathrm{~d}$, ${ }^{3} J_{(\mathrm{HH})} 7.32 \mathrm{~Hz}, 2 \mathrm{H}, \mathrm{H}-2$ ',6'), 7.57-7.52 (m, 1H, H-4'), 7.50-7.46 (m, 2H, H-3',5'), 7.39 (dd, $\left.{ }^{2} J_{(\mathrm{HH})} 8.24 \mathrm{~Hz},{ }^{3} J_{(\mathrm{HH})} 1.36 \mathrm{~Hz}, 1 \mathrm{H}, \mathrm{H}-4\right), 7.11\left(\mathrm{dd},{ }^{2} J_{(\mathrm{HH})} 8.28 \mathrm{~Hz},{ }^{3} J_{(\mathrm{HH})} 4.36 \mathrm{~Hz}, 1 \mathrm{H}, \mathrm{H}-5\right)$, $4.80\left(\mathrm{~d},{ }^{3} J_{(\mathrm{HH})} 2.28 \mathrm{~Hz}, 2 \mathrm{H}, \mathrm{O}-\mathrm{CH}_{2}\right), 2.60\left(\mathrm{t},{ }^{3} J_{(\mathrm{HH})} 2.72 \mathrm{~Hz}, 1 \mathrm{H}, \mathrm{CH}\right),{ }^{13} \mathrm{C}$ NMR $(100 \mathrm{MHz}$, $\left.\mathrm{CDCl}_{3}\right): \delta_{\mathrm{c}} 164.6(\mathrm{C}=\mathrm{O}), 143.3(\mathrm{C}-2), 140.7(\mathrm{C}-3), 134.8\left(\mathrm{C}-1^{\prime}\right), 131.9(\mathrm{C}-6), 128.6\left(\mathrm{C}-2^{\prime}, 6^{\prime}\right)$,

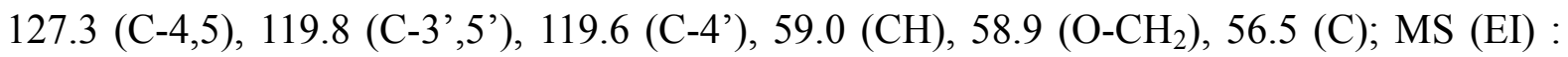
$m / z 253.2\left(\mathrm{M}^{+}+1\right)$; Anal. Calc. for $\mathrm{C}_{15} \mathrm{H}_{12} \mathrm{~N}_{2} \mathrm{O}_{2}$ : C, 71.42; H, 4.79; N, 11.10. Found: C, 71.75; $\mathrm{H}, 4.96 ; \mathrm{N}, 11.33$.

General procedure for synthesis of $\mathbf{6 a - h}$. In a round bottom flask, $\mathrm{Cu}(\mathrm{OAc})_{2} \cdot \mathrm{H}_{2} \mathrm{O}(5$ mol\%), 1,10-phenanthroline monohydrate $(5 \mathrm{~mol} \%)$ and sodium L-ascorbate $(107 \mathrm{mg}, 0.54$ mmol) were added in EtOH: $\mathrm{H}_{2} \mathrm{O}(6: 4,10 \mathrm{~mL})$ and the mioxture stirred for 5 mins at room 
temperature. $N$-(3-(prop-2-ynyloxy)-pyridin-2-yl)benzamide 5 (100 mg, $0.40 \mathrm{mmol}$ ), sodium azide $(76 \mathrm{mg}, 1.17 \mathrm{mmol})$ and the benzyl halide $(0.40 \mathrm{mmol})$ were added to the reaction mixture with stirring at room temperature. Reaction time varied from $10 \mathrm{~min}$ to $24 \mathrm{~h}$ for various benzyl halides. After completion of the reaction (monitored by TLC), ice cold water was added to the reaction mixture till the product precipitated, it was filtered off and washed with cold water. The crude product was then dried under vacuum. The crude product was purified by column chromatography using AcOEt:MeOH (98:2) as eluent to give compounds 6a-h.

$\mathrm{N}$-(3-((1-benzyl-1H-1,2,3-triazol-4-yl)methoxy)pyridin-2-yl)benzamide (6a). Yield: $55 \%$; mp 120-122 ${ }^{\circ} \mathrm{C} ;{ }^{1} \mathrm{H}$ NMR (400 MHz, $\mathrm{CDCl}_{3}$ ): $\delta_{\text {н }} 8.50$ (bs, $\left.1 \mathrm{H}, \mathrm{NH}\right), 8.13\left(\mathrm{dd},{ }^{2} J_{(\mathrm{HH})} 5.04 \mathrm{~Hz}\right.$, $\left.{ }^{3} J_{(\mathrm{HH})} 1.36 \mathrm{~Hz}, 1 \mathrm{H}, \mathrm{H}-6\right), 7.89-7.87$ (m, 2H, H-C2',6'), 7.57-7.53 (m, 2H, H-4', 5'), 7.48-7.44 (m, 2H, H-3',5'), 7.42 (dd, $\left.{ }^{2} J_{(\mathrm{HH})} 8.24 \mathrm{~Hz},{ }^{3} J_{(\mathrm{HH})} 1.36 \mathrm{~Hz}, 1 \mathrm{H}, \mathrm{H}-5\right), 7.35$ (t, ${ }^{3} J_{(\mathrm{HH})} 2.76 \mathrm{~Hz}$, 3H, H-2" ",4"”,6"'), 7.23-7.21 (m, 2H, H-3" ,5"'), 7.09 (dd, ${ }^{2} J_{(\mathrm{HH})} 8.24 \mathrm{~Hz},{ }^{3} J_{(\mathrm{HH})} 5.04 \mathrm{~Hz}$, $1 \mathrm{H}, \mathrm{H}-4$ ') 5.50 (s, 2H, O-CH $), 5.29\left(\mathrm{~s}, 2 \mathrm{H}, \mathrm{N}-\mathrm{CH}_{2}\right) ;{ }^{13} \mathrm{C} \mathrm{NMR}\left(100 \mathrm{MHz}, \mathrm{CDCl}_{3}\right): \delta_{\mathrm{c}} 164.8$ $(\mathrm{C}=\mathrm{O}), 144.6$ (C-2), 143.2 (C-3), 142.1 (C-1',C-4”), 140.4 (C-1"”), 134.6 (C-5”), 131.9 (C6), 129.1 (C-2',6'), 128.8 (C-4'), 128.6 (C-3',5'), 128.0 (C-2'”,6'”), 127.4 (C-4), 123.1 (C-

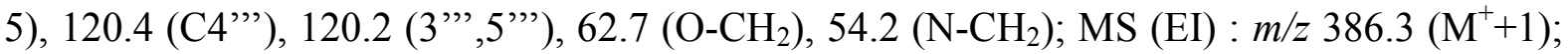
Anal. Calc. for $\mathrm{C}_{22} \mathrm{H}_{19} \mathrm{~N}_{5} \mathrm{O}_{2}: \mathrm{C}, 68.56 ; \mathrm{H}, 4.97 ; \mathrm{N}, 18.17$. Found: $\mathrm{C}, 68.73 ; \mathrm{H}, 5.23 ; \mathrm{N}, 18.34$.

$\mathbf{N}$-(3-((1-(4-Chlorobenzyl)-1H-1,2,3-triazol-4-yl)methoxy)pyridin-2-yl)benzamide (6b). Yield: $65 \%$; mp 163-164 ${ }^{\circ} \mathrm{C} ;{ }^{1} \mathrm{H}$ NMR $\left(400 \mathrm{MHz}, \mathrm{CDCl}_{3}\right): \delta_{\text {н }} 8.49$ (bs, $\left.1 \mathrm{H}, \mathrm{NH}\right), 8.12$ (d, $\left.{ }^{3} J_{(\mathrm{HH})} 3.20 \mathrm{~Hz}, 1 \mathrm{H}, \mathrm{H}-6\right), 7.88$ (d, ${ }^{3} J_{(\mathrm{HH})} 7.32 \mathrm{~Hz}, 2 \mathrm{H}, \mathrm{H}-2$ ', 6'), 7.58 (s, 1H, H-5”), 7.56 (d, $\left.{ }^{3} J_{(\mathrm{HH})} 7.32 \mathrm{~Hz}, 1 \mathrm{H}, \mathrm{H}-4\right), 7.48$ (t, $\left.{ }^{3} J_{(\mathrm{HH})} 7.32 \mathrm{~Hz}, 2 \mathrm{H}, \mathrm{H}-3^{\prime}, 5^{\prime}\right), 7.41$ (d, ${ }^{3} J_{(\mathrm{HH})} 7.76 \mathrm{~Hz}, 1 \mathrm{H}, \mathrm{H}-$

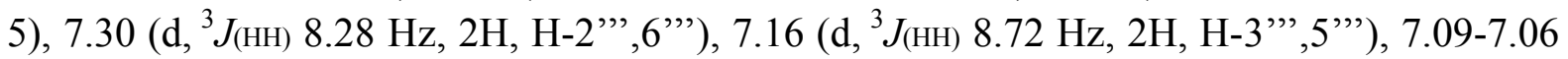
(m, 1H, H-4"'), 5.46 (s, 2H, O-CH $), 5.29\left(\mathrm{~s}, 2 \mathrm{H}, \mathrm{N}-\mathrm{CH}_{2}\right) ;{ }^{13} \mathrm{C} \mathrm{NMR}\left(100 \mathrm{MHz}, \mathrm{CDCl}_{3}\right): \delta_{\mathrm{c}}$ 164.9 (C=O), 144.8 (C-2), 143.5 (C-3), 142.0 (C-1',4”), 140.3 (C-1'”), 134.8 (C-4"'), 134.5 (C-5”), 132.6 (C2'”,6”'), 131.8 (C-6), 129.9 (C-2',6'), 129.0 (C-4'), 128.7 (C-3',5'), 127.4

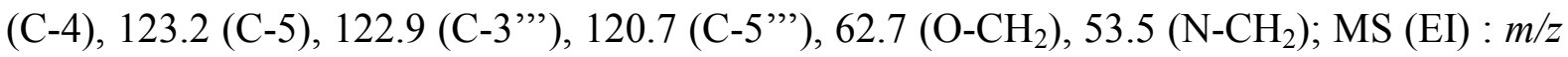
$420.1\left(\mathrm{M}^{+}+1\right)$; Anal. Calc. for $\mathrm{C}_{22} \mathrm{H}_{18} \mathrm{ClN}_{5} \mathrm{O}_{2}$ : C, 62.93; H, 4.32; N, 16.68. Found: C, 62.79; $\mathrm{H}, 4.48 ; \mathrm{N}, 16.59$.

$\mathbf{N}$-(3-((1-(3-Chlorobenzyl)-1 H-1,2,3-triazol-4-yl)methoxy)pyridin-2-yl)benzamide (6c). Yield: $66 \%$; mp 154-157 ${ }^{\circ} \mathrm{C}$; ${ }^{1} \mathrm{H}$ NMR $\left(400 \mathrm{MHz}, \mathrm{CDCl}_{3}\right.$ ): $\delta_{\text {н }} 8.52$ (bs, $1 \mathrm{H}, \mathrm{NH}$ ), 8.13 (dd, $\left.{ }^{2} J_{(\mathrm{HH})} 5.04 \mathrm{~Hz},{ }^{3} J_{(\mathrm{HH})} 1.36 \mathrm{~Hz}, 1 \mathrm{H}, \mathrm{H}-6\right), 7.90$ (d, ${ }^{3} J_{(\mathrm{HH})} 6.88 \mathrm{~Hz}, 2 \mathrm{H}, \mathrm{H}-2$ ', 6'), 7.61 (s, 1H, H5'), 7.57-7.54 (m, 1H, H-4), 7.48 (t, ${ }^{3} J_{(\mathrm{HH})} 7.36 \mathrm{~Hz}, 2 \mathrm{H}, \mathrm{H}-3$ ',5'), 7.41 (dd, ${ }^{2} J_{(\mathrm{HH})} 8.24 \mathrm{~Hz}$, $\left.{ }^{3} J_{(\mathrm{HH})} 1.40 \mathrm{~Hz}, 1 \mathrm{H}, \mathrm{H}-5\right), 7.33-7.28$ (m, 2H, H-2"',6"'), 7.22 (t, ${ }^{3} J_{(\mathrm{HH})} 1.84 \mathrm{~Hz}, 1 \mathrm{H}, \mathrm{H}-4$ '), $7.10\left(\mathrm{dd},{ }^{2} J_{(\mathrm{HH})} 8.24 \mathrm{~Hz},{ }^{3} J_{(\mathrm{HH})} 5.04 \mathrm{~Hz}, 2 \mathrm{H}, \mathrm{H}-4\right.$ '", 5 "' $), 5.47$ (s, 2H, O-CH $)_{2}, 5.31$ (s, 2H, N$\left.\mathrm{CH}_{2}\right) ;{ }^{13} \mathrm{C}$ NMR $\left(100 \mathrm{MHz}, \mathrm{CDCl}_{3}\right): \delta_{\mathrm{c}} 164.9(\mathrm{C}=\mathrm{O}), 144.8(\mathrm{C}-2), 143.5(\mathrm{C}-3), 142.1(\mathrm{C}-$ 1',4"), 140.7 (C-1"'), 140.3 (C-2"”), 136.1 (C-3"'), 134.9 (C-6"'), 134.6 (C-5"), 131.8 (C6), 131.0 (C-2',6'), 129.0 (C-4'), 128.4 (C-3',5'), 127.4 (C-4), 123.0 (C-5), 121.2 (C-4'”), 120.0 (C-5"'), $62.7\left(\mathrm{O}^{\left.-\mathrm{CH}_{2}\right)}, 53.5\left(\mathrm{~N}-\mathrm{CH}_{2}\right)\right.$; $\mathrm{MS}(\mathrm{EI}): \mathrm{m} / \mathrm{z} 420.1\left(\mathrm{M}^{+}+1\right)$; Anal. Calc. for $\mathrm{C}_{22} \mathrm{H}_{18} \mathrm{ClN}_{5} \mathrm{O}_{2}$ : C, 62.93; H, 4.32; N, 16.68. Found: C, 62.71; H, 4.53; N, 16.47.

$\mathbf{N}$-(3-((1-(2-Chlorobenzyl)-1 H-1,2,3-triazol-4-yl)methoxy)pyridin-2-yl)benzamide (6d). Yield: 64\%; mp 125-126 ${ }^{\circ} \mathrm{C}$; ${ }^{1} \mathrm{H}$ NMR (400 MHz, $\mathrm{CDCl}_{3}$ ): $\delta_{\text {н }} 8.53$ (bs, $1 \mathrm{H}, \mathrm{NH}$ ), 8.13 (dd, $\left.{ }^{2} J_{(\mathrm{HH})} 5.04 \mathrm{~Hz},{ }^{3} J_{(\mathrm{HH})} 1.40 \mathrm{~Hz}, 1 \mathrm{H}, \mathrm{H}-6\right), 7.90$ (d, ${ }^{3} J_{(\mathrm{HH})} 7.32 \mathrm{~Hz}, 2 \mathrm{H}, \mathrm{H}-2$ ', 6'), 7.66 (s, 1H, H5"), 7.57-7.54 (m, 1H, H-5), 7.48 (t, ${ }^{3} J_{(\mathrm{HH})} 2.76 \mathrm{~Hz}, 2 \mathrm{H}, \mathrm{H}-3$ ',5'), 7.43-7.39 (m, 2H, H- 
4"”,6"'), 7.32-7.28 (m, 1H, H-3"”), 7.25-7.21 (m, 1H, H-5"”), 7.19 (dd, ${ }^{2} J_{(\mathrm{HH})} 7.76 \mathrm{~Hz},{ }^{3} J_{(\mathrm{HH})}$ $1.84 \mathrm{~Hz}, 1 \mathrm{H}, \mathrm{H}-4), 7.09$ (dd, ${ }^{2} J_{(\mathrm{HH})} 8.24 \mathrm{~Hz},{ }^{3} J_{(\mathrm{HH})} 5.04 \mathrm{~Hz}, 1 \mathrm{H}, \mathrm{H}-4$ ') 5.64 (s, 2H, O-CH $)_{2}$, $5.30\left(\mathrm{~s}, 2 \mathrm{H}, \mathrm{N}-\mathrm{CH}_{2}\right) ;{ }^{13} \mathrm{C}$ NMR $\left(100 \mathrm{MHz}, \mathrm{CDCl}_{3}\right): \delta_{\mathrm{c}} 164.9(\mathrm{C}=\mathrm{O}), 144.6(\mathrm{C}-2), 143.1(\mathrm{C}-3)$, 142.1 (C-1',4”), 140.5 (C-1'”), 134.6 (C-5”), 133.5 (C-2'”), 131.9 (C-6), 130.5 (C-2',6'), 130.4 (C-3"'), 129.9 (C-4'), 128.6 (C-3',5'), 127.6 (C-6”'), 127.4 (C-4), 123.4 (C-5), 120.4 (C-4"”), 120.3 (C-5"'), $62.7\left(\mathrm{O}^{\left.-\mathrm{CH}_{2}\right),} 51.5\left(\mathrm{~N}^{\left.-\mathrm{CH}_{2}\right)}\right.\right.$; MS (EI) : m/z $420.1\left(\mathrm{M}^{+}+1\right)$; Anal. Calc. for $\mathrm{C}_{22} \mathrm{H}_{18} \mathrm{ClN}_{5} \mathrm{O}_{2}$ : C, 62.93; H, 4.32; N, 16.68. Found: $\mathrm{C}, 62.59 ; \mathrm{H}, 4.57 ; \mathrm{N}, 16.85$.

$\mathrm{N}$-(3-((1-(4-Fluorobenzyl)-1 H-1,2,3-triazol-4-yl)methoxy)pyridin-2-yl)benzamide (6e). Yield: $82 \%$; mp 165-168 ${ }^{\circ} \mathrm{C}$; ${ }^{1} \mathrm{H}$ NMR $\left(400 \mathrm{MHz}, \mathrm{CDCl}_{3}\right): \delta_{\text {н }} 8.52$ (bs, $\left.1 \mathrm{H}, \mathrm{NH}\right), 8.12(\mathrm{~d}$, $\left.{ }^{3} J_{(\mathrm{HH})} 4.56 \mathrm{~Hz}, 1 \mathrm{H}, \mathrm{H}-6\right), 7.88$ (d, ${ }^{3} J_{(\mathrm{HH})} 6.88 \mathrm{~Hz}, 2 \mathrm{H}, \mathrm{H}-2$ ', 6'), 7.57 (s, 1H, H-5"), 7.56-7.54 (m, 1H, H-4), 7.47 (t, $\left.{ }^{3} J_{(\mathrm{HH})} 7.32 \mathrm{~Hz}, 2 \mathrm{H}, \mathrm{H}-3^{\prime}, 5^{\prime}\right), 7.41$ (dd, ${ }^{2} J_{(\mathrm{HH})} 7.80 \mathrm{~Hz},{ }^{3} J_{(\mathrm{HH})} 1.36 \mathrm{~Hz}$, 1H, H-5), 7.22-7.19 (m, 2H, H-2"', 6"”), 7.09 (dd, ${ }^{2} J_{(\mathrm{HH})} 8.28 \mathrm{~Hz},{ }^{3} J_{(\mathrm{HH})} 5.04 \mathrm{~Hz}, 1 \mathrm{H}, \mathrm{H}-4$ '), 7.03-6.98 (m, 2H, H-3"”,5"'), $5.46\left(\mathrm{~s}, 2 \mathrm{H}, \mathrm{O}-\mathrm{CH}_{2}\right), 5.29\left(\mathrm{~s}, 2 \mathrm{H}, \mathrm{N}-\mathrm{CH}_{2}\right) ;{ }^{13} \mathrm{C}$ NMR (100 $\left.\mathrm{MHz}, \mathrm{CDCl}_{3}\right): \delta_{\mathrm{c}} 164.9(\mathrm{C}=\mathrm{O}), 144.8$ (C-2), 143.4 (C-3), 142.0 (C-4”), 140.6 (C-1'), 140.3 (C-1"'), 134.5 (C-5"), 131.8 (C-6), 130.5 (C-2',6'), 130.0 (C2'”), 129.3 (C-4'), 128.9 (C3',5'), 128.3 (C-6"'), 127.4 (C-4), 123.2 (C-5), 120.0, 119.9 (C-3"'), 115.5, 115.3 (C-5'”), $62.7\left(\mathrm{O}-\mathrm{CH}_{2}\right), 53.4\left(\mathrm{~N}-\mathrm{CH}_{2}\right)$; $\mathrm{MS}(\mathrm{EI}): \mathrm{m} / z 404.2\left(\mathrm{M}^{+}+1\right)$; Anal. Calc. for $\mathrm{C}_{22} \mathrm{H}_{18} \mathrm{FN}_{5} \mathrm{O}_{2}: \mathrm{C}$, 65.50; H, 4.50; N, 17.36. Found: C, 65.38; H, 4.33; N, 17.51 .

$\mathbf{N}$-(3-((1-(2-Fluorobenzyl)-1 H-1,2,3-triazol-4-yl)methoxy)pyridin-2-yl)benzamide (6f). Yield: 78\%; mp 132-134 ${ }^{\circ} \mathrm{C}$; ${ }^{1} \mathrm{H}$ NMR $\left(400 \mathrm{MHz}, \mathrm{CDCl}_{3}\right): \delta_{\text {н }} 8.53(\mathrm{bs}, 1 \mathrm{H}, \mathrm{NH}), 8.13(\mathrm{~d}$, $\left.{ }^{3} J_{(\mathrm{HH})} 4.56 \mathrm{~Hz}, 1 \mathrm{H}, \mathrm{H}-4\right), 7.90$ (d, ${ }^{3} J_{(\mathrm{HH})} 6.84 \mathrm{~Hz}, 2 \mathrm{H}, \mathrm{H}-2^{\prime}, 6$ ') 7.66 (s, 1H, H-5 ") ) 7.57-7.53 (m, 1H, H-4), 7.48 (t, $\left.{ }^{3} J_{(\mathrm{HH})} 7.32 \mathrm{~Hz}, 2 \mathrm{H}, \mathrm{H}-3,5^{\prime}\right), 7.42$ (dd, ${ }^{2} J_{(\mathrm{HH})} 8.24 \mathrm{~Hz},{ }^{3} J_{(\mathrm{HH})} 1.36 \mathrm{~Hz}$, 1H, H-5), 7.37-7.32 (m, 1H, H-6"'), 7.24 (dd, ${ }^{2} J_{(\mathrm{HH})} 7.80 \mathrm{~Hz},{ }^{3} J_{(\mathrm{HH})} 1.84 \mathrm{~Hz}, 1 \mathrm{H}, \mathrm{H}-4$ '), 7.137.05 (m, 3H, H-3"', ,4"',5"'), 5.56 (s, 2H, O-CH $), 5.29\left(\mathrm{~s}, 2 \mathrm{H}, \mathrm{N}-\mathrm{CH}_{2}\right) ;{ }^{13} \mathrm{C} \mathrm{NMR}(100 \mathrm{MHz}$, $\left.\mathrm{CDCl}_{3}\right): \delta_{\mathrm{c}} 164.8(\mathrm{C}=\mathrm{O}), 144.6(\mathrm{C}-2), 143.2(\mathrm{C}-3), 142.1$ (C-4"'), 140.7 (C-1'), 140.4 (C-1'”), 134.7 (C-5”), 131.7 (C-6), 129.9 (C-2',6'), 128.9, 128.8 (C-2'”), 128.4 (C-4'), 127.4 (C3',5'), 124.2 (C-6"”), 123.2 (C-4), 121.0 (C-4"'), 120.5 (C-3"”), 119.8, 119.7 (C-5"'), 62.7 $\left(\mathrm{O}-\mathrm{CH}_{2}\right), 47.8\left(\mathrm{~N}-\mathrm{CH}_{2}\right)$; MS (EI) : $m / z$ 404.2 $\left(\mathrm{M}^{+}+1\right)$; Anal. Calc. for $\mathrm{C}_{22} \mathrm{H}_{18} \mathrm{FN}_{5} \mathrm{O}_{2}$ : C, 65.50; H, 4.50; N, 17.36. Found: C, 65.33; H, 4.68; N, 17.18 .

$\boldsymbol{N}$-(3-((1-(2-Nitrobenzyl)-1 $\boldsymbol{H}$-1,2,3-triazol-4-yl)methoxy)pyridin-2-yl)benzamide (6g). Yield: $60 \%$; mp 119-123 ${ }^{\circ} \mathrm{C}$; ${ }^{1} \mathrm{H}$ NMR (400 MHz, $\mathrm{CDCl}_{3}$ ): $\delta_{\text {н }} 8.56$ (bs, $\left.1 \mathrm{H}, \mathrm{NH}\right), 8.14$ (dd, $\left.{ }^{2} J_{(\mathrm{HH})} 7.76 \mathrm{~Hz},{ }^{3} J_{(\mathrm{HH})} 1.32 \mathrm{~Hz}, 2 \mathrm{H}, \mathrm{H}-6,4{ }^{\prime \prime \prime}\right), 7.90$ (d, ${ }^{3} J_{(\mathrm{HH})} 7.36 \mathrm{~Hz}, 2 \mathrm{H}, \mathrm{H}-2$ ',6'), 7.84 (s, 1H, H-5"), 7.58-7.50 (m, 3H, H-4,3',5'), 7.48 (m, 3H, H-5,4',6"'), 7.10-7.05 (m, 2H, H-3"', ,'”), $5.90\left(\mathrm{~s}, 2 \mathrm{H}, \mathrm{O}-\mathrm{CH}_{2}\right), 5.34\left(\mathrm{~s}, 2 \mathrm{H}, \mathrm{N}-\mathrm{CH}_{2}\right) ;{ }^{13} \mathrm{C} \mathrm{NMR}\left(100 \mathrm{MHz}, \mathrm{CDCl}_{3}\right): \delta_{\mathrm{c}} 164.8(\mathrm{C}=\mathrm{O})$, 147.3 (C-2"'), 144.6 (C-2), 142.1 (C-3,4”), 140.2 (C-1'), 140.0 (C-1"”), 134.5 (C-5”), 134.2 (C-3"'), 131.8 (C-6), 131.1 (C2',6'), 130.0 (C-2'”'), 129.8 (C-6"'), 129.0 (C-4'), 128.3 (C3'), 127.4 (C-5'), 125.5 (C-4), 125.2 (C-4"”), 124.3 (C-4"”), 120.1 (C-3"'), 62.7 (O-CH ${ }_{2}$, $51.0\left(\mathrm{~N}-\mathrm{CH}_{2}\right)$; MS (EI) : $m / z 431.4\left(\mathrm{M}^{+}+1\right)$; Anal. Calc. for $\mathrm{C}_{22} \mathrm{H}_{18} \mathrm{~N}_{6} \mathrm{O}_{40}$ : C, 61.39; $\mathrm{H}, 4.22$; N, 19.53. Found: C, 61.47; H, 4.39; N, 19.76.

$\mathrm{N}$-(3-((1-Allyl-1H-1,2,3-triazol-4-yl)methoxy)pyridin-2-yl)benzamide (6h). Yield: 55\%; mp 117-120 ${ }^{\circ} \mathrm{C} ;{ }^{1} \mathrm{H}$ NMR (400 MHz, $\left.\mathrm{CDCl}_{3}\right): \delta_{\mathrm{H}} 8.58(\mathrm{bs}, 1 \mathrm{H}, \mathrm{NH}), 8.13\left(\mathrm{~d},{ }^{3} J_{(\mathrm{HH})} 4.12 \mathrm{~Hz}\right.$, 1H, H-6), 7.92-7.90 (m, 2H, H-2',6'), 7.65 (s, 1H, H-5"), 7.57-7.53 (m, 1H, H-5), 7.49-7.46 (m, 2H, H-3', 5'), 7.43 (dd, $\left.{ }^{2} J_{(\mathrm{HH})} 7.80 \mathrm{~Hz},{ }^{3} J_{(\mathrm{HH})} 1.36 \mathrm{~Hz}, 1 \mathrm{H}, \mathrm{H}-4\right), 7.09$ (dd, ${ }^{2} J_{(\mathrm{HH})} 8.28 \mathrm{~Hz}$, 


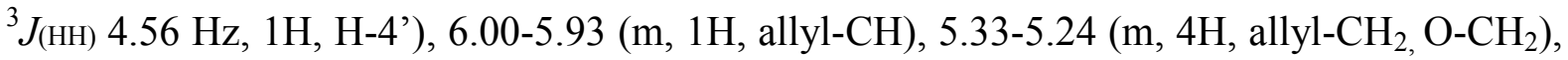
4.96-4.94 (m, 2H, N-CH $\left.) ;{ }^{13} \mathrm{C} \mathrm{NMR} \mathrm{(100} \mathrm{MHz,} \mathrm{CDCl}_{3}\right): \delta_{\mathrm{c}} 164.9(\mathrm{C}=\mathrm{O}), 144.8(\mathrm{C}-2), 143.1$ (C-3), 142.1 (C-4”), 134.6 (C-5”), 131.9 (C-6), 130.7 (C-2',6'), 128.6 (C-4'), 127.4 (C-

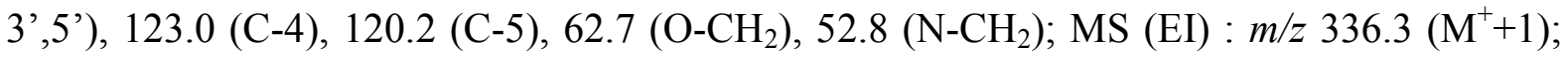
Anal. Calc. for $\mathrm{C}_{18} \mathrm{H}_{17} \mathrm{~N}_{5} \mathrm{O}_{2}$ : C, 64.47; H, 5.11; N, 20.88. Found: C, 64.39; H, 5.39; N, 20.65.

Synthesis of $\boldsymbol{N}$-(3-hydroxypyridin-2-yl)-3-phenylacrylamide 8. In a $100 \mathrm{ml}$ round bottom flask, cinnamic acid $(2.6 \mathrm{~g}, 18 \mathrm{mmol})$ was stirred in $\mathrm{CH}_{2} \mathrm{Cl}_{2}(50 \mathrm{~mL})$ at $0{ }^{\circ} \mathrm{C}$ in the presence of HOBt (5.4 g, $40 \mathrm{mmol})$, EDC (6.2 g, $40 \mathrm{mmol})$ and DIPEA (2.5 g, $20 \mathrm{mmol})$. 2-Amino-3hydroxy pyridine $\mathbf{I}(2 \mathrm{~g}, 18 \mathrm{mmol})$ was added to the above suspension and the miture stirred at room temperature for $12 \mathrm{~h}$. After completion of the reaction, the reaction mixture was extracted with water. The organic layer was separated, dried over $\mathrm{Na}_{2} \mathrm{SO}_{4}$, filtered and concentrated to get crude product. Purification with column chromatography resulted in compound $\mathbf{8}$ as a yellow solid.

$N$-(3-hydroxypyridin-2-yl)-3-phenylacrylamide (8). Yield: 80\%; mp 145-147 ${ }^{\circ} \mathrm{C} ;{ }^{1} \mathrm{H}$ NMR $\left(400 \mathrm{MHz}, \mathrm{CDCl}_{3}\right): \delta_{\text {н }} 10.90(\mathrm{bs}, 1 \mathrm{H}, \mathrm{OH}), 10.20$ (bs, $\left.1 \mathrm{H}, \mathrm{NH}\right), 7.94\left(\mathrm{~d},{ }^{3} J_{(\mathrm{HH})} 4.12 \mathrm{~Hz}, 1 \mathrm{H}\right.$, H-6), 7.88 (d, $\left.J_{(\mathrm{HH})} 15.12 \mathrm{~Hz}, 1 \mathrm{H}, \mathrm{H}-5\right), 7.52-7.50$ (m, 2H, H-2',6'), 7.44-7.38 (m, 4H, CH, H4,3',5'), $7.19\left(\mathrm{dd},{ }^{2} J_{(\mathrm{HH})} 7.80 \mathrm{~Hz},{ }^{3} J_{(\mathrm{HH})} 4.56 \mathrm{~Hz}, 1 \mathrm{H}, \mathrm{H}-4\right.$ '), $6.75\left(\mathrm{~d},{ }^{3} J_{(\mathrm{HH})} 15.60 \mathrm{~Hz}, 1 \mathrm{H}, \mathrm{CH}\right)$; ${ }^{13} \mathrm{C}$ NMR $\left(100 \mathrm{MHz}, \mathrm{CDCl}_{3}\right): \delta_{\mathrm{c}} 166.3(\mathrm{C}=\mathrm{O}), 145.4(\mathrm{C}-2), 145.1(\mathrm{C}-3), 140.5(\mathrm{C}-1$ ') 138.2 (CH), 133.9 (CH), 131.0 (C-6), 130.3 (C-2'), 129.3 (C-6'), 128.6 (C-4'), 128.4 (C-3'), 128.0 (C-5'), 118.5 (C-4), 117.4 (C-5). MS (EI) : $m / z 241.1\left(\mathrm{M}^{+}+1\right)$.

Synthesis of 3-phenyl- $N$-prop-2-ynyl- $N$-(3-(prop-2-ynyloxy)pyridin-2-yl)acrylamide (9) and 3-phenyl- $N$-(3-(prop-2-ynyloxy)pyridin-2-yl)acrylamide (10). $N$-(3-Hydroxy-pyridin2-yl)-3-phenyl-acrylamide 8 (2 g, $10 \mathrm{mmol})$ was treated with propargyl bromide $(1.17 \mathrm{~g}, 10$ $\mathrm{mmol})$ in the presence of $\mathrm{K}_{2} \mathrm{CO}_{3}(2 \mathrm{~g}, 15 \mathrm{mmol})$ and $\mathrm{TBAHSO}_{4}(0.05 \mathrm{mmol})$ using acetonitrile as solvent. When the reaction was carried out at room temperature for $8 \mathrm{~h}$, the resulting compounds were obtained as yellow solids of 3-phenyl- $N$-(3-(prop-2-ynyloxy)-pyridin-2yl)acrylamide (10) and 3-phenyl- $N$-prop-2-ynyl- $N$-(3-(prop-2-ynyloxy)-pyridin-2yl)acrylamide (9) in 55\% and $10 \%$ yields respectively. Acetonitrile was removed under vacuum, extracted with $\mathrm{CHCl}_{3}$ and water, the organic layer separated and dried over $\mathrm{Na}_{2} \mathrm{SO}_{4}$ to get the crude product. Column chromatography was required in order to get the corresponding compounds 9 and 10. When this reaction was carried out at reflux temperature for $8 \mathrm{~h}$, it resulted in formation of yellowish liquid of 3-phenyl- $N$-prop-2-ynyl- $N$-(3-(prop-2-ynyloxy)pyridin-2-yl)acrylamide (9) in 60\% yield with traces of compound $\mathbf{1 0}$.

3-Phenyl- $N$-prop-2-ynyl- $N$-(3-(prop-2-ynyloxy)-pyridin-2-yl)acrylamide (9). Yield: $60 \%$; ${ }^{1} \mathrm{H}$ NMR $\left(400 \mathrm{MHz}, \mathrm{CDCl}_{3}\right): \delta_{\text {н }} 8.72\left(\mathrm{dd},{ }^{2} J_{(\mathrm{HH})} 4.60 \mathrm{~Hz},{ }^{3} J_{(\mathrm{HH})} 1.40 \mathrm{~Hz}, 1 \mathrm{H}, \mathrm{H}-6\right), 7.74(\mathrm{~d}$, $\left.{ }^{3} J_{(\mathrm{HH})} 15.12 \mathrm{~Hz}, 1 \mathrm{H}, \mathrm{H}-5\right), 7.53\left(\mathrm{dd},{ }^{2} J_{(\mathrm{HH})} 8.24 \mathrm{~Hz},{ }^{3} J_{(\mathrm{HH})} 1.36 \mathrm{~Hz}, 1 \mathrm{H}, \mathrm{H}-2\right.$ '), 7.38 (dd, ${ }^{2} J_{(\mathrm{HH})}$ $8.24 \mathrm{~Hz},{ }^{3} J_{(\mathrm{HH})} 4.56 \mathrm{~Hz}, 1 \mathrm{H}, \mathrm{H}-6$ ') $7.34\left(\mathrm{dd},{ }^{2} J_{(\mathrm{HH})} 5.96 \mathrm{~Hz},{ }^{3} J_{(\mathrm{HH})} 2.20 \mathrm{~Hz}, 2 \mathrm{H}, \mathrm{H}-4,4\right.$ '), 7.28 (d, ${ }^{3} J_{(\mathrm{HH})} 2.28 \mathrm{~Hz}, 3 \mathrm{H}, \mathrm{CH}, \mathrm{H}-3$ ', $\left.5^{\prime}\right), 6.23\left(\mathrm{~d},{ }^{3} J_{(\mathrm{HH})} 15.12 \mathrm{~Hz}, 1 \mathrm{H}, \mathrm{CH}\right), 4.74\left(\mathrm{~d},{ }^{3} J_{(\mathrm{HH})} 2.15\right.$ $\left.\mathrm{Hz}, 2 \mathrm{H}, \mathrm{O}-\mathrm{CH}_{2}\right), 4.71\left(\mathrm{~d},{ }^{3} J_{(\mathrm{HH})} 2.28 \mathrm{~Hz}, 2 \mathrm{H}, \mathrm{N}-\mathrm{CH}_{2}\right), 2.40\left(\mathrm{t},{ }^{3} J_{(\mathrm{HH})} 2.30 \mathrm{~Hz}, 1 \mathrm{H}, \mathrm{CH}\right), 2.14$ (d, $\left.{ }^{3} J_{(\mathrm{HH})} 2.72 \mathrm{~Hz}, 1 \mathrm{H}, \mathrm{CH}\right)$. MS (EI) : $m / z 317.1\left(\mathrm{M}^{+}+1\right)$; Anal. Calc. for $\mathrm{C}_{20} \mathrm{H}_{16} \mathrm{~N}_{2} \mathrm{O}_{2}: \mathrm{C}$, 75.93; H, 5.10; N, 8.86. Found: C, 75.67; H, 4.99; N, 8.93.

3-Phenyl- $N$-(3-(prop-2-ynyloxy)pyridin-2-yl)acrylamide (10). Yield: 55\%; mp 129-131 ${ }^{\circ} \mathrm{C}$; ${ }^{1} \mathrm{H}$ NMR (400 MHz, CDCl $): \delta_{\text {н }} 8.10\left(\mathrm{~d},{ }^{3} J_{(\mathrm{HH})} 4.56 \mathrm{~Hz}, 2 \mathrm{H}, \mathrm{NH}, \mathrm{H}-6\right), 7.85\left(\mathrm{~d},{ }^{3} J_{(\mathrm{HH})}\right.$ 
16.04 Hz, 1H, H-5), 7.60-7.57 (m, 2H, H-2',6'), 7.41-7.34 (m, 4H, CH,H-4,3',5'), 7.32 (dd, ${ }^{2} J_{(\mathrm{HH})} 8.24 \mathrm{~Hz},{ }^{3} J_{(\mathrm{HH})} 1.36 \mathrm{~Hz}, 1 \mathrm{H}, \mathrm{H}-4$ '), $7.05\left(\mathrm{dd},{ }^{2} J_{(\mathrm{HH})} 8.24 \mathrm{~Hz},{ }^{3} J_{(\mathrm{HH})} 5.04 \mathrm{~Hz}, 1 \mathrm{H}, \mathrm{CH}\right)$, $4.79\left(\mathrm{~d},{ }^{3} J_{(\mathrm{HH})} 2.28 \mathrm{~Hz}, 2 \mathrm{H}, \mathrm{O}-\mathrm{CH}_{2}\right), 2.60\left(\mathrm{t},{ }^{3} J_{(\mathrm{HH})} 2.30 \mathrm{~Hz}, 1 \mathrm{H}, \mathrm{CH}\right) ;{ }^{13} \mathrm{C}$ NMR $(100 \mathrm{MHz}$, $\left.\mathrm{CDCl}_{3}\right): \delta_{\mathrm{c}} 164.8(\mathrm{C}=\mathrm{O}), 143.3(\mathrm{C}-2), 142.3(\mathrm{C}-3), 142.2(\mathrm{C}-1$ ') $, 140.1(\mathrm{CH}), 134.8(\mathrm{CH})$, 129.8 (C-6), 128.7 (C-2',6'), 128.1 (C-4'), 120.6 (C-3',5'), 119.2 (C-4), 119.1 (C-5), 56.3 (O$\left.\mathrm{CH}_{2}\right)$; MS (EI) : m/z 279.3 (M+1); Anal. Calc. for $\mathrm{C}_{17} \mathrm{H}_{14} \mathrm{~N}_{2} \mathrm{O}_{2}$ : C, 73.37; H, 5.07; N, 10.07 . Found: C, 73.60; H, 5.32; N, 10.29.

General procedure for synthesis of 11a-g. In a round bottom flask, $\mathrm{Cu}(\mathrm{OAc})_{2} \cdot \mathrm{H}_{2} \mathrm{O}(5$ mol\%), 1,10-phenanthroline monohydrate (5 mol\%) and sodium L-ascorbate (107 $\mathrm{mg}, 0.47$ mmol) were added in $\mathrm{EtOH}: \mathrm{H}_{2} \mathrm{O}(6: 4,10 \mathrm{~mL})$ and the mixture stirred for 5 mins at room temperature. Compound 10 (100 mg, $0.35 \mathrm{mmol})$, sodium azide (76 mg, $1.02 \mathrm{mmol})$ and the corresponding benzyl halide $(0.40 \mathrm{mmol})$ were added to the reaction mixture and it was stirred at room temperature. Reaction time varied from 8 to $20 \mathrm{~h}$ for various benzyl halides. After completion of reaction (monitored by TLC), ice cold water was added to reaction mixture till the product precipitated out. It was filtered off and washed with cold water. The crude product was purified by column chromatography using AcOEt:MeOH (98:2) as eluent to get compound 11a-g.

$\mathrm{N}$-[3-(1-Benzyl-1H-[1,2,3]triazol-4-ylmethoxy)pyridin-2-yl]-3-phenyl-acrylamide (11a). Yield: $50 \%$; mp 125-126 ${ }^{\circ} \mathrm{C}$; ${ }^{1} \mathrm{H}$ NMR $\left(400 \mathrm{MHz}, \mathrm{CDCl}_{3}\right.$ ): $\delta_{\text {н }} 8.11$ (bs, $\left.1 \mathrm{H}, \mathrm{NH}\right), 8.06$ (dd, $\left.{ }^{2} J_{(\mathrm{HH})} 4.56 \mathrm{~Hz},{ }^{3} J_{(\mathrm{HH})} 0.92 \mathrm{~Hz}, 1 \mathrm{H}, \mathrm{H}-6\right), 7.82\left(\mathrm{~d},{ }^{3} J_{(\mathrm{HH})} 15.6 \mathrm{~Hz}, 1 \mathrm{H}, \mathrm{H}-5\right), 7.59-7.57$ (m, 3H, H-5',2',6'), 7.41-7.37 (m, 4H, CH,H-3',4',5'), 7.36-7.32 (m, 4H, H-2'",3'",5"',6"'), 7.317.27 (m, 2H, H-4,4"'), $7.03\left(\mathrm{dd},{ }^{2} J_{(\mathrm{HH})} 8.28 \mathrm{~Hz},{ }^{3} J_{(\mathrm{HH})} 5.04 \mathrm{~Hz}, 1 \mathrm{H}, \mathrm{CH}\right), 5.54\left(\mathrm{~s}, 2 \mathrm{H}, \mathrm{N}-\mathrm{CH}_{2}\right)$, $5.26\left(\mathrm{~s}, 2 \mathrm{H}, \mathrm{N}-\mathrm{CH}_{2}\right) ;{ }^{13} \mathrm{C} \mathrm{NMR}\left(100 \mathrm{MHz}, \mathrm{CDCl}_{3}\right): \delta_{\mathrm{c}} 164.8(\mathrm{C}=\mathrm{O}), 143.2(\mathrm{C}-2), 143.0(\mathrm{C}-3)$, 142.1 (C-1,4”), 139.9 (C-1"'), 134.9 (C-5”), $134.0(\mathrm{CH}), 129.9(\mathrm{CH}), 129.2$ (C-6), 128.9 (C2',6'), 128.7 (C-4'), 128.1 (C-3',5'), 127.9 (C-2'”,6'”), 127.1 (C-4), 123.2 (C-5), 120.6 (C-

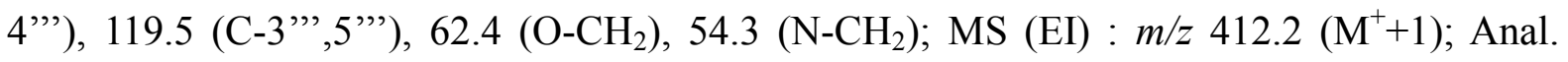
Calc. for $\mathrm{C}_{24} \mathrm{H}_{21} \mathrm{~N}_{5} \mathrm{O}_{2}$ : C, 70.06; H, 5.14; N, 17.02. Found: C, 70.27; H, 4.82; N, 17.37.

$\mathbf{N}$-\{3-[1-(4-Chloro-benzyl)-1 $\boldsymbol{H}$-[1,2,3]triazol-4-ylmethoxy]-pyridin-2-yl\}-3-phenylacrylamide (11b). Yield: 58\%; mp 160-162 ${ }^{\circ} \mathrm{C} ;{ }^{1} \mathrm{H}$ NMR (400 MHz, $\mathrm{CDCl}_{3}$ ): $\delta_{\mathrm{H}} 8.07$ (bs, $1 \mathrm{H}$, $\mathrm{NH}), 8.06\left(\mathrm{dd},{ }^{2} J_{(\mathrm{HH})} 4.80 \mathrm{~Hz},{ }^{3} J_{(\mathrm{HH})} 1.60 \mathrm{~Hz}, 1 \mathrm{H}, \mathrm{H}-6\right), 7.82\left(\mathrm{~d},{ }^{3} J_{(\mathrm{HH})} 15.56 \mathrm{~Hz}, 1 \mathrm{H}, \mathrm{H}-5\right)$, 7.60-7.59 (m, 2H, H-2',6'), 7.57 (s, 1H, H-5'), 7.41-7.39 (m, 2H, CH, H-4'), 7.38 (d, ${ }^{3} J_{(\mathrm{HH})}$ $1.36 \mathrm{~Hz}, 2 \mathrm{H}, \mathrm{H}-5$ ',6'), 7.37-7.34 (m, 2H, H-2"',6"'”), 7.33 (t, $\left.{ }^{3} J_{(\mathrm{HH})} 2.28 \mathrm{~Hz}, 1 \mathrm{H}, \mathrm{H}-4\right), 7.23-$ 7.21 (m, 2H, H-3"', 5"'), 7.04 (dd, $\left.{ }^{2} J_{(\mathrm{HH})} 8.24 \mathrm{~Hz},{ }^{3} J_{(\mathrm{HH})} 5.04 \mathrm{~Hz}, 1 \mathrm{H}, \mathrm{CH}\right), 5.51$ (s, 2H, O$\left.\mathrm{CH}_{2}\right), 5.26\left(\mathrm{~s}, 2 \mathrm{H}, \mathrm{N}-\mathrm{CH}_{2}\right) ;{ }^{13} \mathrm{C} \mathrm{NMR}\left(100 \mathrm{MHz}, \mathrm{CDCl}_{3}\right): \delta_{\mathrm{c}} 164.8(\mathrm{C}=\mathrm{O}), 143.3(\mathrm{C}-2), 143.2$ (C-3), 142.1 (C-1',4"), 140.0 (C-1"”), 135.0 (C-4"'), 134.9 (C-5"), 134.1 (CH), 132.5 (C2"',6”"), 130.0 (CH), 129.9 (C-2',6'), 129.5 (C-4'), 129.4 (C-3'), 128.8 (C-5'), 128.1 (C-4),

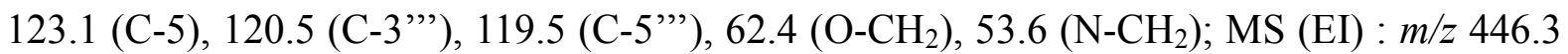
$\left(\mathrm{M}^{+}+1\right)$; Anal. Calc. for $\mathrm{C}_{24} \mathrm{H}_{20} \mathrm{ClN}_{5} \mathrm{O}_{2}$ : C, 64.65; H, 4.52; N, 15.71. Found: $\mathrm{C}, 64.37 ; \mathrm{H}$, $4.73 ; \mathrm{N}, 15.82$.

\section{$\mathrm{N}$-\{3-[1-(3-Chloro-benzyl)-1 $\boldsymbol{H}$-[1,2,3]triazol-4-ylmethoxy]pyridin-2-yl\}-3-phenyl-}

acrylamide (11c). Yield: $57 \%$; $\mathrm{mp} 161-163{ }^{\circ} \mathrm{C} ;{ }^{1} \mathrm{H}$ NMR (400 MHz, $\left.\mathrm{CDCl}_{3}\right): \delta_{\mathrm{H}} 8.30$ (bs, $1 \mathrm{H}$, $\mathrm{NH}), 8.06\left(\mathrm{dd},{ }^{2} J_{(\mathrm{HH})} 5.04 \mathrm{~Hz},{ }^{3} J_{(\mathrm{HH})} 0.92 \mathrm{~Hz}, 1 \mathrm{H}, \mathrm{H}-6\right), 7.82$ (d, $\left.{ }^{3} J_{(\mathrm{HH})} 16.04 \mathrm{~Hz}, 1 \mathrm{H}, \mathrm{H}-5\right)$, 7.66 (s, 1H, H-5”), 7.59-7.56 (m, 2H, H-2',6'), 7.40-7.39 (m, 2H, CH, H-4'), 7.38 (d, ${ }^{3} J_{(\mathrm{HH})}$ 
$1.84 \mathrm{~Hz}, 3 \mathrm{H}, \mathrm{H}-4,5^{\prime}, 6$ ') $7.32-7.30$ (m, 2H, H-2"',6"'), 7.28 (s, 1H, H-2"'), 7.15 (d, ${ }^{3} J_{(\mathrm{HH})}$ $6.88 \mathrm{~Hz}, 1 \mathrm{H}, \mathrm{H}-4$ "') 7.06 (dd, $\left.{ }^{2} J_{(\mathrm{HH})} 7.80 \mathrm{~Hz},{ }^{3} J_{(\mathrm{HH})} 5.04 \mathrm{~Hz}, 1 \mathrm{H}, \mathrm{CH}\right), 5.51$ (s, 2H, O-CH $)_{2}$, $5.29\left(\mathrm{~s}, 2 \mathrm{H}, \mathrm{N}-\mathrm{CH}_{2}\right) ;{ }^{13} \mathrm{C} \mathrm{NMR}\left(100 \mathrm{MHz}, \mathrm{CDCl}_{3}\right): \delta_{\mathrm{c}} 164.8(\mathrm{C}=\mathrm{O}), 145.9(\mathrm{C}-2), 143.6(\mathrm{C}-3)$, 143.4 (C-1',4"), 143.3 (C-1"”), 142.2 (C-2"”), 139.8 (C-3"”), 136.0 (C-6"'), 135.0 (CH), 134.8 (C-5”), 130.4 (CH), 129.9 (C-6), 129.1 (C-2',6'), 128.8 (C-4'), 128.7 (C-3'), 128.2 (C5'), 128.1 (C-4), 123.3 (C-5), 120.5 (C-4"”), 119.8 (C-5"'), $62.5\left(\mathrm{O}-\mathrm{CH}_{2}\right), 53.6\left(\mathrm{~N}^{-\mathrm{CH}_{2}}\right)$; MS (EI) : $m / z 446.3\left(\mathrm{M}^{+}+1\right)$; Anal. Calc. for $\mathrm{C}_{24} \mathrm{H}_{20} \mathrm{ClN}_{5} \mathrm{O}_{2}$ : C, 64.65; H, 4.52; N, 15.71. Found: C, 64.23; H, 4.29; N, 15.90 .

$\boldsymbol{N}$-\{3-[1-(2-Chloro-benzyl)-1 $\boldsymbol{H}$-[1,2,3]triazol-4-ylmethoxy]pyridin-2-yl\}-3-phenylacrylamide (11d). Yield: 56\%; mp 128-129 ${ }^{\circ} \mathrm{C} ;{ }^{1} \mathrm{H}$ NMR (400 MHz, $\mathrm{CDCl}_{3}$ ): $\delta_{\text {н }} 8.09$ (bs, $1 \mathrm{H}$, $\mathrm{NH}), 8.07\left(\mathrm{dd},{ }^{2} J_{(\mathrm{HH})} 5.04 \mathrm{~Hz},{ }^{3} J_{(\mathrm{HH})} 0.92 \mathrm{~Hz}, 1 \mathrm{H}, \mathrm{H}-6\right), 7.59\left(\mathrm{dd},{ }^{2} J_{(\mathrm{HH})} 7.80 \mathrm{~Hz},{ }^{3} J_{(\mathrm{HH})} 1.36\right.$ $\mathrm{Hz}, 1 \mathrm{H}, \mathrm{H}-5$ ), 7.43 (s, 1H, H-5'), 7.41-7.39 (m, 2H, H-2',6'), 7.38 (d, ${ }^{3} J_{(\mathrm{HH})} 1.36 \mathrm{~Hz}, 2 \mathrm{H}, \mathrm{H}-$ 3', ', ), $7.33\left(\mathrm{dd},{ }^{2} J_{(\mathrm{HH})} 6.88 \mathrm{~Hz},{ }^{3} J_{(\mathrm{HH})} 2.28 \mathrm{~Hz}, 1 \mathrm{H}, \mathrm{CH}\right), 7.29-7.27$ (m, 2H, H-4,4'), 7.26 (d, ${ }^{3} J_{(\mathrm{HH})} 1.36 \mathrm{~Hz}, 1 \mathrm{H}, \mathrm{H}-3$ '”'), 7.25-7.23 (m, 2H, H-5"',6"'), 7.16-7.11 (m, 1H, H-4"'), 7.04 (dd, $\left.{ }^{2} J_{(\mathrm{HH})} 7.80 \mathrm{~Hz},{ }^{3} J_{(\mathrm{HH})} 5.04 \mathrm{~Hz}, 1 \mathrm{H}, \mathrm{CH}\right), 5.68\left(\mathrm{~s}, 2 \mathrm{H}, \mathrm{O}-\mathrm{CH}_{2}\right), 5.27\left(\mathrm{~s}, 2 \mathrm{H}, \mathrm{N}_{-} \mathrm{CH}_{2}\right) ;{ }^{13} \mathrm{C} \mathrm{NMR}$ $\left(100 \mathrm{MHz}, \mathrm{CDCl}_{3}\right): \delta_{\mathrm{c}} 164.8(\mathrm{C}=\mathrm{O}), 144.5(\mathrm{C}-2), 143.2$ (C-3), 142.9 (C-1',4”), $140.0(\mathrm{C}-$ 1"'), 135.1 (CH), 134.9 (C-5"), 133.2 (C-2"'), 131.9 (CH), 130.5 (C-6), 130.4 (C-2',6'), 130.0 (C-3'”), 129.8 (C-4'), 129.2 (C-3'), 128.7 (C-5'), 128.1 (C-6"'), 127.6 (C-4), 123.5 (C5), 120.6 (C-4"”), 119.5 (C-5"'), $62.4\left(\mathrm{O}-\mathrm{CH}_{2}\right), 51.6\left(\mathrm{~N}-\mathrm{CH}_{2}\right)$; $\mathrm{MS}$ (EI) : $m / z 446.3\left(\mathrm{M}^{+}+1\right)$; Anal. Calc. for $\mathrm{C}_{24} \mathrm{H}_{20} \mathrm{ClN}_{5} \mathrm{O}_{2}$ : C, 64.65; H, 4.52; N, 15.71. Found: C, 64.73; H, 4.17; N, 15.33 .

$\mathrm{N}$-\{3-[1-(4-Fluoro-benzyl)-1 $\mathrm{H}$-[1,2,3]triazol-4-ylmethoxy]-pyridin-2-yl\}-3-phenylcrylamide (11e). Yield: $58 \%$; mp $169-172{ }^{\circ} \mathrm{C} ;{ }^{1} \mathrm{H}$ NMR $\left(400 \mathrm{MHz}, \mathrm{CDCl}_{3}\right): \delta_{\mathrm{H}} 8.20(\mathrm{bs}, 1 \mathrm{H}$, $\mathrm{NH}), 8.05\left(\mathrm{~d},{ }^{3} J_{(\mathrm{HH})} 4.56 \mathrm{~Hz}, 1 \mathrm{H}, \mathrm{H}-6\right), 7.81\left(\mathrm{~d},{ }^{3} J_{(\mathrm{HH})} 15.56 \mathrm{~Hz}, 1 \mathrm{H}, \mathrm{H}-5\right), 7.62$ (s, 1H, H-5"'), 7.58-7.56 (m, 2H, H-2',6'), 7.39 (d, ${ }^{3} J_{(\mathrm{HH})} 1.84 \mathrm{~Hz}, 2 \mathrm{H}, \mathrm{H}-3^{\prime}, 5^{\prime}$ ), 7.38-7.36 (m, 2H, CH, H-4), 7.29-7.27 (m, 3H, H-2'”, 6"'), 7.07 (d, ${ }^{3} J_{(\mathrm{HH})} 8.68 \mathrm{~Hz}, 2 \mathrm{H}, \mathrm{H}-3$ "', 5 '”), 7.06 (d, ${ }^{3} J_{(\mathrm{HH})} 2.28 \mathrm{~Hz}$, $1 \mathrm{H}, \mathrm{CH}), 5.50\left(\mathrm{~s}, 2 \mathrm{H}, \mathrm{O}-\mathrm{CH}_{2}\right), 5.26\left(\mathrm{~s}, 2 \mathrm{H}, \mathrm{N}-\mathrm{CH}_{2}\right) ;{ }^{13} \mathrm{C} \mathrm{NMR}\left(100 \mathrm{MHz}, \mathrm{CDCl}_{3}\right): \delta_{\mathrm{c}} 164.1$ $(\mathrm{C}=\mathrm{O}), 143.4$ (C-2), 143.2 (C-3), 142.1 (C-4”), 139.8 (C-1'), 135.3 (CH), 134.8 (C-5"), $130.1(\mathrm{CH}), 130.0$ (C-6), 129.9 (C-2'), 129.8 (C-6'), 128.7 (C-2'”), 128.1 (C-4'), 126.5 (C3',5'), 123.1 (C-6"'), 120.6 (C-4), 119.6 (C-5), 116.3 (C-3"”), 114.0 (C-5"'), 62.5 (O-CH ${ }_{2}$ ), $53.5\left(\mathrm{~N}-\mathrm{CH}_{2}\right)$; MS (EI) : $m / z$ 430.2 $\left(\mathrm{M}^{+}+1\right)$; Anal. Calc. for $\mathrm{C}_{24} \mathrm{H}_{20} \mathrm{FN}_{5} \mathrm{O}_{2}$ : C, 67.12; $\mathrm{H}, 4.69$; N, 16.31. Found: C, 66.83; H, 4.53; N, 16.43.

\section{$\mathrm{N}$-\{3-[1-(2-Fluoro-benzyl)-1 $\boldsymbol{H}$-[1,2,3]triazol-4-ylmethoxy]pyridin-2-yl\}-3-phenyl-}

acrylamide (11f). Yield: 55\%; mp 138-140 ${ }^{\circ} \mathrm{C} ;{ }^{1} \mathrm{H}$ NMR (400 MHz, $\left.\mathrm{CDCl}_{3}\right): \delta_{\mathrm{н}} 8.16$ (bs, $1 \mathrm{H}$, $\mathrm{NH}), 8.05$ (s, 1H, H-6), 7.81 (d, $\left.{ }^{3} J_{(\mathrm{HH})} 15.60 \mathrm{~Hz}, 1 \mathrm{H}, \mathrm{H}-5\right), 7.70$ (s, 1H, H-5'), 7.58 (d, ${ }^{3} J_{(\mathrm{HH})}$ $2.40 \mathrm{~Hz}, 1 \mathrm{H}, \mathrm{H}-2^{\prime}$ ), 7.37-7.28 (m, 7H, CH, H-4,3',4',5',6',3'”, ), 7.16-7.07 (m, 4H, CH, H$4 ",, 5 ", 6 "$ ") $5.60\left(\mathrm{~s}, 2 \mathrm{H}, \mathrm{O}-\mathrm{CH}_{2}\right), 5.26\left(\mathrm{~s}, 2 \mathrm{H}, \mathrm{N}-\mathrm{CH}_{2}\right) ;{ }^{13} \mathrm{C} \mathrm{NMR}\left(100 \mathrm{MHz}, \mathrm{CDCl}_{3}\right): \delta_{\mathrm{c}}$ $161.7(\mathrm{C}=\mathrm{O}), 144.5$ (C-2), 143.1 (C-3), 143.0 (C-4”), 140.3 (C-1',1'”), 135.6 (CH), 134.9 (C-5'), 131.1 (CH), 131.0 (C-6), 130.6 (C-2',6'), 129.9 (C-2'”), 128.7 (C-4'), 128.1 (C-3'), 124.9 (C-5'), 123.4 (C-6"'), 121.5 (C-4), 121.3 (C-4"”), 119.5 (C-3"”), 115.7 (C-5"'), 62.4 $\left(\mathrm{O}-\mathrm{CH}_{2}\right), 47.9\left(\mathrm{~N}-\mathrm{CH}_{2}\right)$; MS (EI) : $m / z$ 430.2 $\left(\mathrm{M}^{+}+1\right)$; Anal. Calc. for $\mathrm{C}_{24} \mathrm{H}_{20} \mathrm{FN}_{5} \mathrm{O}_{2}: \mathrm{C}, 67.12$; H, 4.69; N, 16.31. Found: C, 67.43; H, 4.53; N, 16.56 . 
$\boldsymbol{N}$-[3-(1-Allyl-1H-[1,2,3]triazol-4-ylmethoxy)pyridin-2-yl]-3-phenyl-acrylamide (11g). Yield: $45 \%$; mp 120-122 ${ }^{\circ} \mathrm{C}$; ${ }^{1} \mathrm{H}$ NMR $\left(400 \mathrm{MHz}, \mathrm{CDCl}_{3}\right.$ ): $\delta_{\text {н }} 8.45$ (bs, $1 \mathrm{H}, \mathrm{NH}$ ), 8.29 (dd, $\left.{ }^{2} J_{(\mathrm{HH})} 7.76 \mathrm{~Hz},{ }^{3} J_{(\mathrm{HH})} 1.80 \mathrm{~Hz}, 1 \mathrm{H}, \mathrm{H}-6\right), 8.12$ (s, 1H, H-5), 8.07-8.03 (m, 1H, H-2'), 7.82 (s, $1 \mathrm{H}, \mathrm{H}-5$ '”), 7.81-7.77 (m, 3H, H-3',5',6'), 7.68 (dd, ${ }^{2} J_{(\mathrm{HH})} 8.28 \mathrm{~Hz},{ }^{3} J_{(\mathrm{HH})} 4.60 \mathrm{~Hz}, 1 \mathrm{H}, \mathrm{H}-4$ '), 7.60-7.57 (m, 1H, CH), 7.42-7.37 (m, 2H, CH, H-4), 6.10-5.87 (m, 1H, allyl-CH), 5.57-5.42 (m, 1H, allyl-CH), 5.37-5.31 (m, 3H, allyl-CH, O-CH $\mathrm{CH}_{2}, 5.01-4.91\left(\mathrm{~m}, 2 \mathrm{H}, \mathrm{N}-\mathrm{CH}_{2}\right) ;{ }^{13} \mathrm{C} \mathrm{NMR}$ (100 MHz, $\left.\mathrm{CDCl}_{3}\right): \delta_{\mathrm{c}} 161.9(\mathrm{C}=\mathrm{O}), 144.6(\mathrm{C}-2), 143.3(\mathrm{C}-3), 143.1(\mathrm{C}-4 ”), 140.0(\mathrm{CH})$, 135.0 (C-5”), 131.2 (CH), 130.8 (C-6), 130.0 (C-2',6'), 128.8 (C-4'), 128.3 (C-3',5'), 123.6 (C-4), 120.8 (C-5), $62.6\left(\mathrm{O}-\mathrm{CH}_{2}\right), 48.0\left(\mathrm{~N}_{-} \mathrm{CH}_{2}\right)$; MS (EI) : $\mathrm{m} / \mathrm{z} 362.2\left(\mathrm{M}^{+}+1\right)$; Anal. Calc. for $\mathrm{C}_{20} \mathrm{H}_{19} \mathrm{~N}_{5} \mathrm{O}_{2}$ : C, 66.47; H, 5.30; N, 19.38. Found: C, 66.14; H, 5.11; N, 19.52 .

\section{Supporting Information}

Supporting information (Experimental details, ${ }^{1} \mathrm{H}$ and ${ }^{13} \mathrm{C}$ NMR spectra for the compounds 35, 6a-h, 8-10 and 11a-g), associated with this article can be found, in the online version.

\section{Acknowledgements}

We thank Department of Science and Technology, New Delhi (SR/FT/CS-40/2010) for the research grant. We also thank SAI Labs, Thapar University, Patiala for recording NMR spectra and Punjab University, Chandigarh for recording mass spectra.

\section{References}

1. Dadiboyena, S.; Nefzi, A. Eur. J. Med. Chem. 2011, 46, 5258. http://dx.doi.org/10.1016/j.ejmech.2011.09.016

2. Pitt, W. R.; Parry, D. M.; Perry, B. G. Groom, C. R. J. Med. Chem. 2009, 52, 2952. http://dx.doi.org/10.1021/jm801513z

3. Mohan, D. C.; Sarang, N. B.; Adimurthy, S. Tetrahedron Lett. 2013, 54, 6077. http://dx.doi.org/10.1016/j.tetlet.2013.08.112

4. Fesenko, A. A.; Shutalev, A. D. Tetrahedron Lett. 2012, 53, 6261. http://dx.doi.org/10.1016/j.tetlet.2012.09.022

5. Spencer, J.; Patel, H.; Callear, S. K.; Coles, S. J.; Deadman, J. J. Tetrahedron Lett. 2011, 52,5905 .

http://dx.doi.org/10.1016/j.tetlet.2011.07.147

6. Pagadala, R.; Maddila, S.; Moodley, V.; van Zyl, W. E.; Jonnalagadda, S. B. Tetrahedron Lett. 2014, 55, 4006. http://dx.doi.org/10.1016/j.tetlet.2014.05.089

7. Behbehani, H.; Ibrahim, H. M. Tetrahedron, 2013, 69, 10535. http://dx.doi.org/10.1016/j.tet.2013.10.061 
8. Xie, W.; Xie, S.; Zhou, Y.; Tang, X.; Liu, J.; Yang, W.; Qiu. M. Eur. J. Med. Chem. 2014, $81,22$.

http://dx.doi.org/10.1016/j.ejmech.2014.05.001

9. Berg, R.; Straub, B. F. Beilstein J. Org. Chem. 2013, 9, 2715.

http://dx.doi.org/10.3762/bjoc.9.308

10. Hein, J. E.; Fokin, V. V. Chem. Soc. Rev., 2010, 39, 1302.

http://dx.doi.org/10.1039/b904091a

11. Meldal, M.; Tornøe, C. W. Chem. Rev. 2008, 108, 2952.

http://dx.doi.org/10.1021/cr0783479

12. Ferreira, S. B.; Sodero, A. C. R.; Cardoso, M. F. C.; Lima, E. S.; Kaiser, C. R.; Silva, F. P.; Ferreira, V. F. J. Med. Chem. 2010, 53, 2364.

http://dx.doi.org/10.1021/jm901265h

13. Yuldasheva, K.; Dzhuraev, A. D.; Makhsumov, A. G.; Amanov, N. Pharm. Chem. J. 1991, 25, 728 .

http://dx.doi.org/10.1007/BF00768987

14. Cho, J. H.; Bernard, D. L.; Sidwell, R. W.; Kern, E. R.; Chu, C. K. J. Med. Chem. 2006, $49,1140$.

http://dx.doi.org/10.1021/jm0509750

15. Giffin, M. J.; Heaslet, H.; Brik, A.; Lin, Y.-C.; Cauvi, G.; Wong, C.-H.; McRee, D. E.; Elder, J. H.; Stout, C. D.; Torbett, B. E. J. Med. Chem. 2008, 51, 6263. http://dx.doi.org/10.1021/jm800149m

16. Reck, F.; Zhou, F.; Girardot, M.; Kern, G.; Eyermann, C. J.; Hales, N. J.; Ramsay, R. R.; Gravestock, M. B. J. Med. Chem. 2005, 48, 499.

http://dx.doi.org/10.1021/jm0400810 